\title{
Recoil and vibration in an archery bow equipped with a multi-rod stabilizer
}

\author{
Igor Zaniewski* \\ Casimir Pulaski Technical University, Radom, Poland
}

Received 14 April 2010

Revised 13 December 2010

\begin{abstract}
The aim of this research is to create a mechanical and mathematical model of a multi-rod stabilizer for the sport archery bow and to analyze its capability to damp disagreeable recoil and vibration of the bow during internal ballistic motion. The research methods are based on the Euler-Bernoulli theory of beam bending, Lagrange equations of the second kind, the Cauchy problem, and the Runge-Kutta method. A mathematical software package was used to analyze the problem. The approach to the problem of sport-bow stabilization in the vertical plane that is proposed in this paper addresses the practical needs both of applied engineering mechanics and of the sport of archery. Numerical results from computer simulation are presented in both tabular and graphical form. The common motion of the string and arrow (internal ballistic motion) is accompanied by intense vibration which is caused by disruption of the static force balance at the moment of string release.
\end{abstract}

Keywords: Archery, bow, vibration, recoil, stabilizer, modeling

\section{Nomenclature}

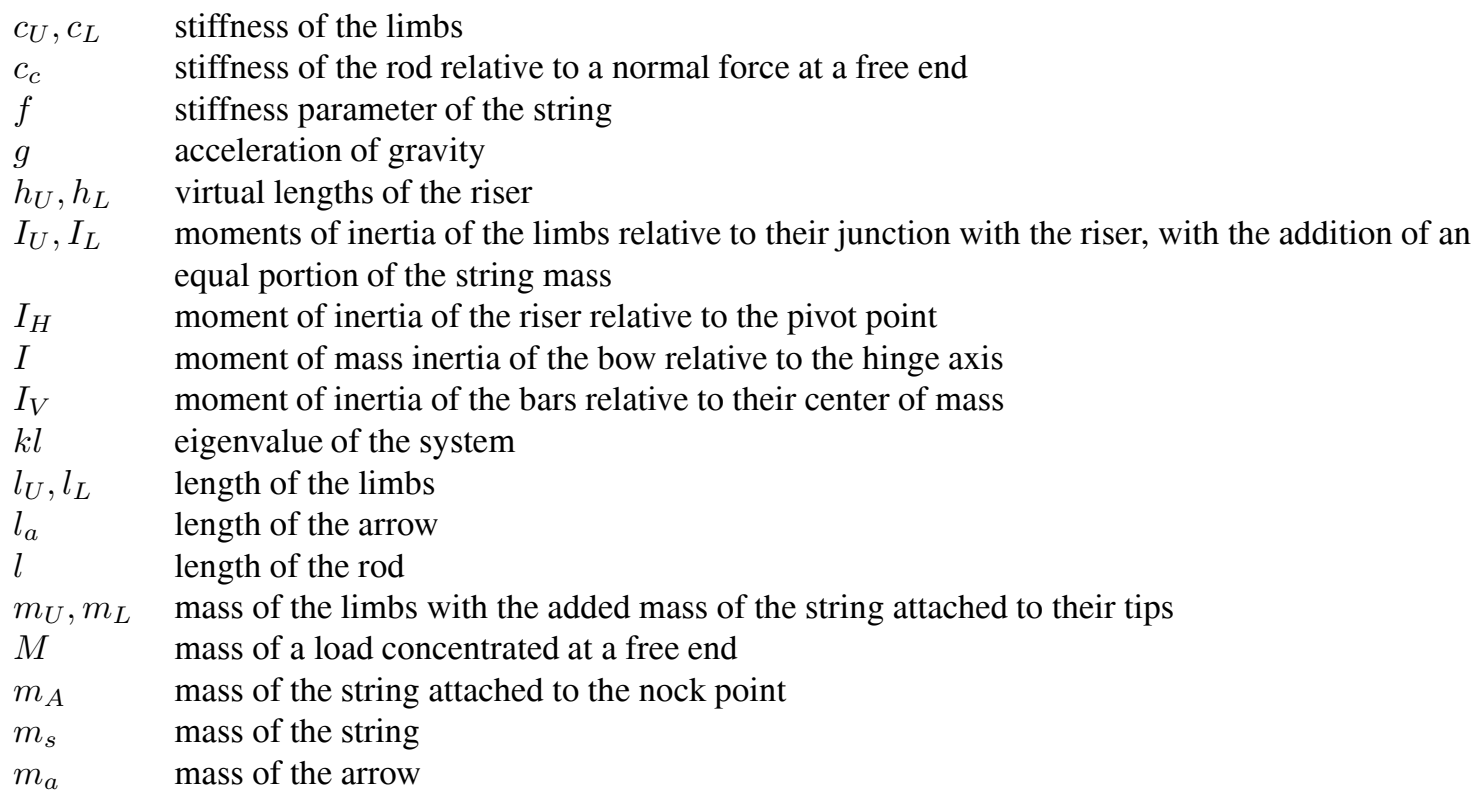

\footnotetext{
*Address for correspondence: Malczewskiego 22, Radom 26-600, Poland. Tel.: +48 48 3618809; Fax: +48 48 3617803; E-mail: izanevsky @onet.eu.
} 


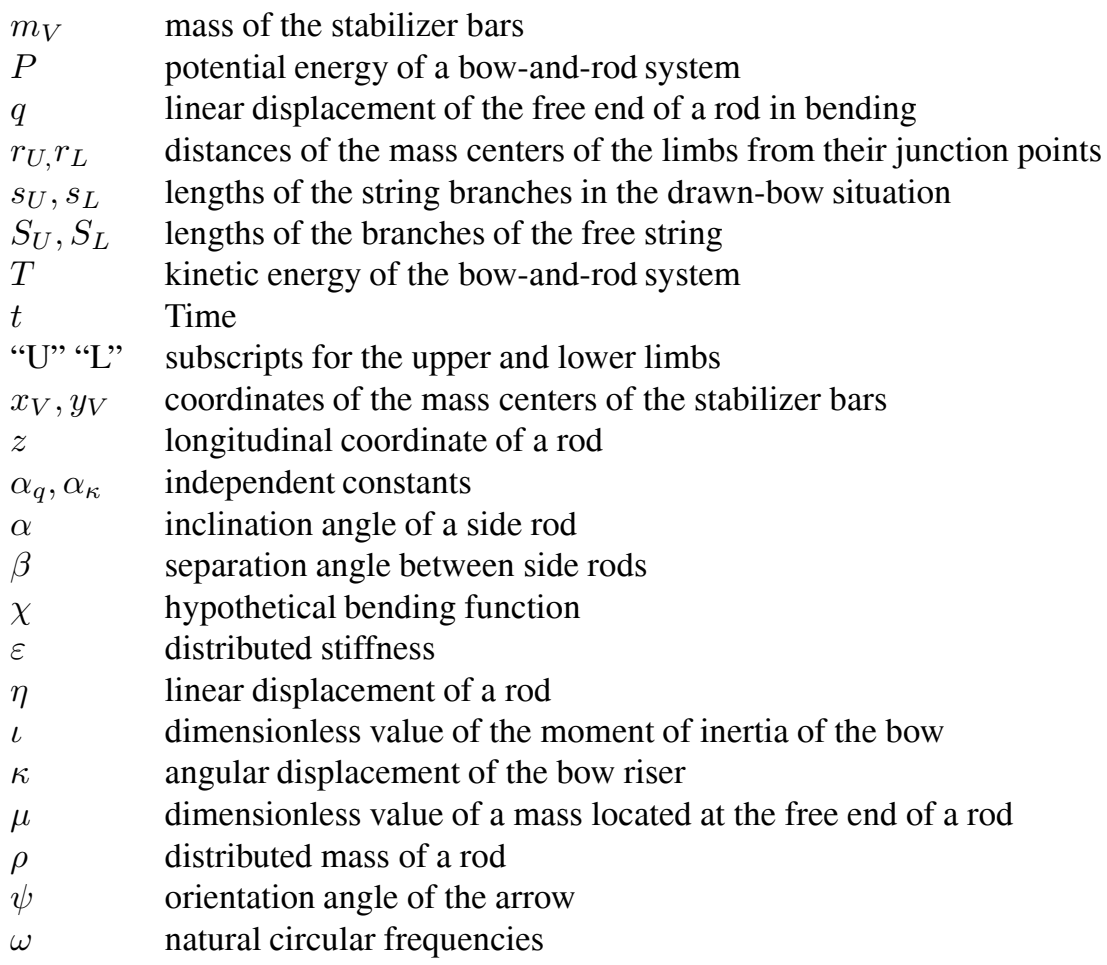

\section{Introduction}

The sharp recoil of the handle after the bowstring has been released and the vibration of the bow after an arrow has been launched from its nock point have a negative effect on results in the sport of archery. Intensive dynamic processes during the joint movement of an arrow and a string result in an unstable extension of the archer's hand to the handle of the bow. This causes a significant dispersion of arrows. Furthermore, the disagreeable sensations of recoil and vibration result in a conditioned counter reflex in the archer just before string release. This causes a disruption of accurate aiming at a target exactly at the moment when an arrow's direction of flight is determined [3].

To reduce this recoil and vibration, modern archery bows are equipped with a special device called a stabilizer. The stabilizer is a system of long and short rods and weights which are mounted on the bow handle [2]. A stabilizer helps the archer to improve the stability of the bow during aiming and during the joint movement of the arrow and string. A stabilizer accumulates and dissipates a part of the kinetic energy involved, reducing the recoil and vibration of the handle.

Commonly, a modern sport-bow stabilizer includes up to five rods mounted on a handle inside the bow [1]. A central rod and two side rods are fastened to an adjustable uni-bar (Appendix A). The central rod is always fastened at the same position perpendicular to the handle. The side rods have an opposite axial direction relative to the central rod and have adjustable space joints. These joints include strong teeth that provide multiple points of adjustment and ensure that the setup does not slip. The uni-bar is fastened to the handle using an extender bar with the same axis as the central rod. The whole system of bars and rods has the main vertical bow plane as its plane of symmetry. Usually, there are one or two additional rods in the stabilizer system. These upper and lower rods are fastened to the handle like cantilever beams in the main plane of the bow and are symmetric relative to the center of the handle (Fig. 1).

The setting and tuning process to adjust the stabilizer parameters to the archer's anthropometric parameters and to his/her style of shooting is based on "trial and error" experimental methods. Because this method requires too much time and effort by archers, a modern training process needs more effective methods and technologies. The development of scientifically based methods to improve stabilizer parameters requires the creation of a mechanical 


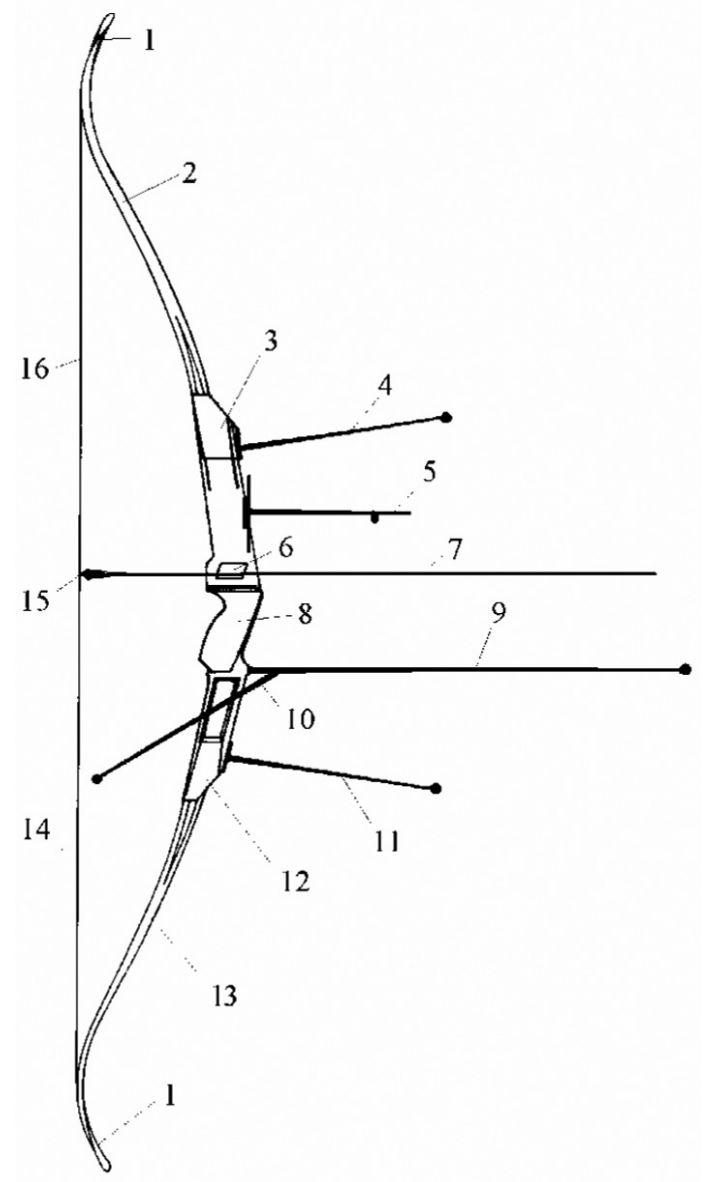

Fig. 1. A modern sport bow in its main plane [6]: 1 - tips; 2, 13 - limbs; 3, 12 - limb-angle setup mechanisms; 4 - upper stabilizer, 5 - sign; 6 arrow rest; 7 - arrow; 8 - handle; 9 - central stabilizer; 10 - side stabilizers; 11 - lower stabilizer; 14, 16 - string branches; 15 - nock point.

and mathematical model of the archery bow stabilizer. This research problem has a substantial theoretical and experimental basis [5-11].

Recoil and vibration in an archery bow equipped with a central-rod stabilizer were studied using mechanical and mathematical modeling methods [5,6]. On this basis, the construction of an advanced three-rod stabilizer was also studied [10]. Because modern sport-bow stabilizers include more than three rods and are equipped with concentrated loads at the free ends of the rods, these models must be improved. The aim of this research is to create a mechanical and mathematical model of a multi-rod stabilizer with concentrated loads at the free ends of the rods and to analyze their capability to damp disagreeable recoil and vibration of a bow during internal ballistic motion.

\section{Basic model of a bow-rod stabilizer}

Each stabilizer rod is modeled as an elastic cantilever beam in the context of Euler-Bernoulli theory because its length is much greater than its cross-sectional diameter. Sport archers stretch a bow with a joint motion of the string and the arrow while trying to maintain a steady body posture. The archer's body mass is significantly greater than that of the bow. Therefore, the point of contact $(O)$ can be assumed to be an immovable pivot point (Fig. 2a). The displacements of points on the handle and stabilizer due to rotation and bending are much less than the length of the rods; therefore, a linear model can be used: 

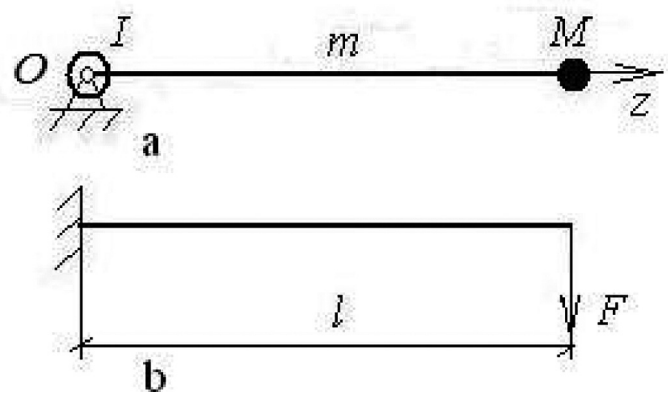

Fig. 2. (a) Bow-and-rod schematic model; (b) cantilever beam loaded at the free end with a concentrated force $F$.

$$
\eta=\kappa z+q \chi
$$

where $\eta$ is the linear displacement of the rod; $z$ is the longitudinal coordinate of the rod; $\kappa$ is the angular displacement of the bow riser (relative to the hinge, p. $O$ ); $q$ is the linear displacement of the free end of the rod in bending; and $\chi$ is a hypothetical bending function. It is assumed that $\kappa$ and $q$ are functions of time, but that $\chi$ is a function of $z$.

It is further assumed that the deformation of the center line during bending of a cylindrical cantilever beam locally loaded at a free end by a force normal to the longitudinal axis (Fig. 2b) can be represented by a hypothetical bending function:

$$
\chi=\frac{1}{2}\left(\frac{z}{l}\right)^{2}\left(3-\frac{z}{l}\right)
$$

where $l$ is the length of the rod. This function satisfies three of four boundary conditions, i.e., zero displacement, zero angle with a normal to the handle at the fixed end, and one of the two dynamic boundary conditions, i.e., zero force moment at the free end.

According to the model, the kinetic $(T)$ and potential $(P)$ energy of a bow-and-rod system can be written as:

$$
T=\frac{1}{2}\left[\int_{0}^{l} \rho\left(\frac{\partial \eta}{\partial t}\right)^{2} d z+I\left(\frac{\partial^{2} \eta}{\partial z \partial t}\right)_{z=0}^{2}+M\left(\frac{\partial \eta}{\partial t}\right)_{z=l}^{2}\right] \text { and } P=\frac{1}{2} \int_{0}^{l} \varepsilon\left(\frac{\partial^{2} \eta}{\partial z^{2}}\right)^{2} d z
$$

where $\rho$ is the distributed mass of the rod; $I$ is the moment of mass inertia of a bow relative to the hinge axis; $M$ is the mass of a load concentrated at a free end (see Fig. 2a); $\varepsilon$ is distributed stiffness; and $t$ is time.

The model was then evaluated for free vibration of the system. Using the Rayleigh-Ritz method, equations for free vibration of a system with a cylindrical $\operatorname{rod}(\rho=$ const and $\varepsilon=$ const $)$ can be obtained as:

$$
\begin{gathered}
\left(\frac{33}{140} m+M\right) \ddot{q}+\left(\frac{11}{40} m+M\right) l \ddot{\kappa}+c q=0 ; \\
{\left[I+\left(\frac{1}{3} m+M\right) l^{2}\right] \ddot{\kappa}+\left(\frac{11}{40} m+M\right) l \ddot{q}=0,}
\end{gathered}
$$

where $m$ is the mass of the rod and $c=\frac{3 \varepsilon}{l^{3}}$ is the stiffness of a cantilever beam with a concentrated force at the free end (see Fig. 2b). Solution of the equations for free vibration yields:

$$
q=\alpha_{q} \sin \omega t ; \quad \kappa=\alpha_{\kappa} \sin \omega t,
$$

where $\alpha_{q}, \alpha_{\kappa}$ are independent constants and $\omega$ represents the natural circular frequency of the system. Substituting the solutions of Eq. (5) into Eq. (4) yields a system of two linear algebraic equations relative to the constants:

$$
\begin{aligned}
& {\left[c-\left(\frac{33}{140} m+M\right) \omega^{2}\right] \alpha_{q}+\left(\frac{11}{40} m+M\right) \omega^{2} l \alpha_{\kappa}=0} \\
& {\left[I+\left(\frac{1}{3} m+M\right) l^{2}\right] \omega^{2} \alpha_{\kappa}+\left(\frac{11}{40} m+M\right) l \omega^{2} \alpha_{q}=0 .}
\end{aligned}
$$




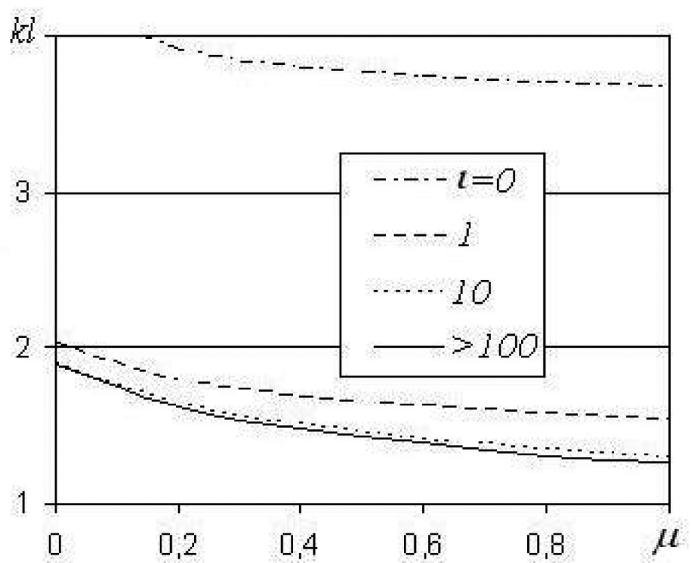

Fig. 3. Main eigenvalue of a bow and stabilizer modeled as a cantilever-rod system.

The two values of $\omega$ for which the determinant of this system of equations is equal to zero are the natural frequencies of the system. One of these is a zero natural frequency that is related to the circular rotation of the bow and rod around the hinge (p. $O$ ). The second value is nonzero and represents coupled bending and rotational vibration. The fourth power of this natural frequency (an eigenvalue) can be represented in a nondimensional form as:

$$
(k l)^{4}=3 /\left[33 / 140+\mu-\frac{(11 / 40+\mu)^{2}}{\iota+1 / 3+\mu}\right],
$$

where $k l=\sqrt[4]{\frac{m l^{3} \omega^{2}}{\varepsilon}}$ is the eigenvalue of the system; $\iota=\frac{I}{m l^{2}}$ is the dimensionless value of the moment of inertia of the bow; and $\mu=\frac{M}{m}$ is the dimensionless value of a mass located at the free end of a rod. Results for the main nonzero eigenvalue for various relationships of mass-inertial parameters are shown in Fig. 3. As expected, the greater the mass at the free end or the moment of mass inertia at the hinge, the lower was the natural frequency.

To verify the stabilizer-rod model based on the hypothetical Eq. (2), a boundary problem using differential equations and boundary conditions was investigated. Because no results have been reported in the open mechanical and mathematical literature for a beam with one end attached to a load and the other end free, this problem is presented here in detail.

Substituting the two expressions for energy in Eq. (3) into a Hamilton functional, $\delta \int_{t_{1}}^{t_{2}}(T-P) d t=0$, a standard procedure yields the corresponding differential equation:

$$
\rho \frac{\partial^{2} \eta}{\partial t^{2}}+\frac{\partial^{2}}{\partial z^{2}}\left(\varepsilon \frac{\partial^{2} \eta}{\partial z^{2}}\right)=0
$$

and boundary conditions

$$
\begin{aligned}
& z=0, \eta=0 ; \varepsilon \frac{\partial^{2} \eta}{\partial z^{2}}=I \frac{\partial^{3} \eta}{\partial z \partial t^{2}} \\
& z=l, \frac{\partial^{2} \eta}{\partial z^{2}}=0 ; \frac{\partial}{\partial z}\left(\varepsilon \frac{\partial^{2} \eta}{\partial z^{2}}\right)=M \frac{\partial^{2} \eta}{\partial t^{2}}
\end{aligned}
$$

Solutions in the form of eigenvalues $(k l)$ were obtained using Krylov functions for a cylindrical rod $(\rho=$ const and $\varepsilon=$ const). They are the roots of the determinant:

$$
\left|\begin{array}{lll}
\iota(k l)^{4} & 2 k l & \iota(k l)^{4} \\
\operatorname{sh}(k l) & \operatorname{ch}(k l)+\cos (k l) & -\sin (k l) \\
\mu k l s h(k l) & \mu k l[c h(k l)-\cos (k l)] & \mu k l \sin (k l) \\
+\operatorname{ch}(k l) & +\operatorname{sh}(k l)-\sin (k l) & -\cos (k l)
\end{array}\right|=0 .
$$


Table 1

Relative error $(\%)$ of the main eigenvalue calculated using the Rayleigh-Ritz method for solution of a boundary problem

\begin{tabular}{ccccccc}
\hline$\iota \backslash \mu$ & 0 & 0.2 & 0.4 & 0.6 & 0.8 & 1 \\
\hline 0 & 9.30 & 11.00 & 11.59 & 11.88 & 12.09 & 12.20 \\
1 & 0.66 & 0.17 & 0.07 & 0.03 & 0.02 & 0.01 \\
10 & 0.72 & 0.21 & 0.10 & 0.06 & 0.04 & 0.02 \\
$>100$ & 0.73 & 0.21 & 0.10 & 0.06 & 0.04 & 0.03 \\
\hline
\end{tabular}

The zero solution of Eq. (7) corresponds with a common rotation of the beam and the load at a fixed end relative to the hinge axis. When $\iota=0, \mu=0$, the result is $k l=0 ; 3,927 ; 7,069 ; 10,210 \ldots$, which are the same as the well-known solutions for the beam with one hinged end, $\frac{\pi(4 i-3)}{4}$, where $i=2,3,4 \ldots$ are the numbers of the natural frequencies. There is no zero solution when $\mu=0, \iota=: k l=1,875 ; 4,694 ; 7,855 \ldots$, which are the same as the well-known solutions for a cantilever beam, $\frac{\pi(2 i-1)}{2}(i=3,4,5 \ldots)$.

For real ratios of the rod and load masses, $(\iota>10 ; \mu<1)$ using the Rayleigh-Ritz method, a very precise estimate (for engineering purposes) of the first natural frequency can be obtained; the relative error of the main eigenvalue is approximately one percent (Table 1). It is interesting to note that the precision of the method increases with the load mass at the free end of the rod. For example, the relative errors for a simple cantilever rod are $0.72-0.73 \%$, but in the case of a cantilever rod with a concentrated load of the same mass (equal to the rod mass) at the free end, the relative error is $0.02-0.03 \%$, i.e., the error decreases more than exponentially with increasing mass. This phenomenon can be explained by the fact that a hypothetical vibration mode presented in a form of a static cantilever rod loaded at one free end is similar to the real mode. The difference between a cantilever rod with a load at one free end and without the load is in the order of the distribution of inertial forces along the rod. The larger the load, the more similar to a static problem is the distribution of inertial forces.

The hypothetical function given in Eq. (2) satisfies three of four boundary conditions. One of two dynamic boundary condition is not satisfied exactly, i.e., a cross-sectional force at the free end. Despite this, the function provides increased precision for the main natural frequency. For example, in the case of a typical sport archery bow with a single-rod stabilizer $(\iota \approx 10 ; \mu \approx 0.2)$, the relative error is approximately $0.21 \%$ (see Table 1$)$ for an eigenvalue and approximately $0.42 \%$ for a natural frequency; because $\omega \sim(k l)^{2}$, the relative error $\delta \omega \sim 2 \delta(k l)$.

\section{Central-rod model}

Movement of the central bar occurs in the vertical plane of symmetry of the bow. Consider a bow with a stabilizer relative to an immovable Cartesian coordinate system, $\xi O \eta$, based on the vertical plane of symmetry of the bow (Fig. 4). In addition, in the same plane, consider a movable Cartesian coordinate system, $x O y$, that is fixed to the riser; the $y$-axis is parallel to the central-rod axis, but oriented in the opposite direction; the $x$-axis is oriented upward. Now consider a displacement of points along the longitudinal axis as a sum of two components: along the axis, $\xi_{c}$, and normal to it, $\eta_{c}$. The longitudinal displacement is the same for all the points of the rod because they depend on the handle angle and the distance of the pivot point $(O)$ from the rod axis. The normal displacement consists of two components: a rotational displacement relative to the pivot point (similar to a longitudinal displacement) and a bending displacement:

$$
\xi_{c}=x_{V} \kappa ; \quad \eta_{c}=\left(z_{c}-y_{V}\right) \kappa+q_{c} \chi,
$$

where $x_{V}, y_{V}$ are the coordinates of a common point of projection to the plane of symmetry of the axes of all three rods; $z_{c}$ is the longitudinal coordinate of the rod; $q_{c}$ is the displacement (as a function of time) of the free end of the rod caused by bending. According to the results of a previous study of the dynamics of a single-offset stabilizer [10], a form of the bending calculation for a horizontal cantilever beam with a concentrated load at the free end can be used Eq. (2).

The kinetic energy of the central rod is:

$$
T_{c}=\frac{1}{2}\left[\int_{0}^{l c} \rho_{c}\left(\dot{\xi}_{c}^{2}+\dot{\eta}_{c}^{2}\right) d z_{c}+M_{c}\left(\dot{\xi}_{c}^{2}+\dot{\eta}_{c}^{2}\right)_{z_{c}=l_{c}}\right]
$$



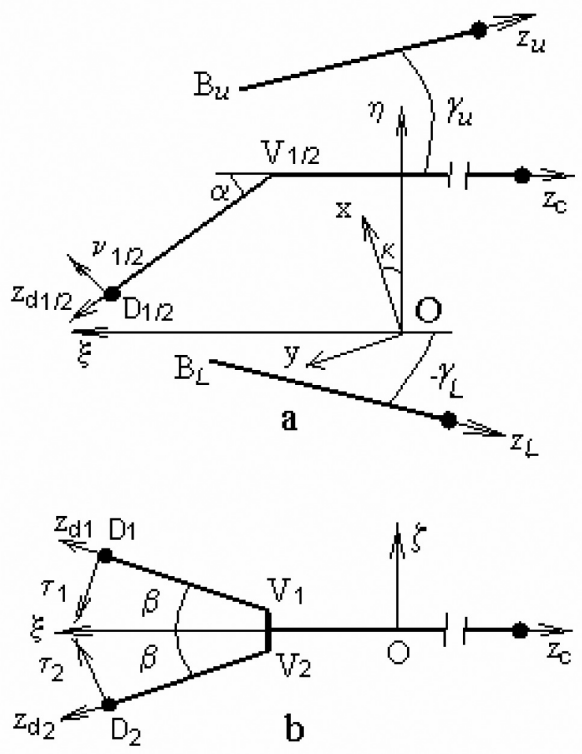

Fig. 4. Schematic model of a multi-rod stabilizer: (a) in the arrow plane and (b).in the transverse plane.

where $l_{c}$ is the length of the central rod; $\rho_{c}$ is the distributed mass of the rod; $M_{c}$ is the mass of a load concentrated at the free end of the rod; and $(\cdot) \equiv\left(\frac{\partial}{\partial t}\right)$ is the sign of the partial derivative with respect to time. Substituting Eqs (8) and (2) into Eq. (9), and after integration and intermediate transformations:

$$
T_{c}=\frac{1}{2} m_{c}\left[\begin{array}{l}
\frac{1}{4}\left(9 J_{4}-6 J_{5}+J_{6}\right) \dot{q}_{c}^{2}+ \\
\left(J_{2} l_{c}^{2}+x_{V}^{2}+y_{V}^{2}-2 y_{V} J_{1} l_{c}\right) \dot{\kappa}^{2}+ \\
{\left[\left(3 J_{3}-J_{4}\right) l_{c}-\left(3 J_{2}-J_{3}\right) y_{V}\right] \dot{q}_{c} \dot{\kappa}}
\end{array}\right]+\frac{1}{2} M_{c}\left\{\begin{array}{l}
{\left[x_{V}^{2}+\left(l_{c}-y_{V}\right)^{2}\right] \dot{\kappa}^{2}} \\
+2\left(l_{c}-y_{V}\right) \dot{\kappa} \dot{q}_{c}+\dot{q}_{c}^{2}
\end{array}\right\},
$$

where $m_{c}=\int_{0}^{l_{c}} \rho_{c} d z_{c}$ is the mass of the rod and

$$
J_{i}=\frac{\int_{0}^{l_{c}} \rho_{c} z_{c}^{i} d z_{c}}{m_{c} l_{c}^{i}}
$$

(Appendix 2). For a cylindrical rod, $J_{i}=\frac{1}{i+1}$.

\section{Side-rod model}

The position of the side rods relative to the bow handle is determined by two angles. The first, the inclination angle $(\alpha)$, is measured between the plane of the side rod and the plane of the central rod (Fig. 4). The second, the separation angle $(\beta)$, is measured between the axis of the rod and the plane of symmetry of the bow. In the schematic diagram, the angle $\alpha$ is presented for the case of parallel side rods $(\beta=0)$. The angular displacement of the handle and the uni-bar $(\kappa)$ is measured counterclockwise from the axis $O \eta$.

The bending displacements of the side rod can be divided into two components. The first $(\nu)$ is a component normal to the rod axis as an upward displacement in the vertical plane when the side rods lie in the same plane as the central rod $(\alpha=0)$ or outside the bow when the side rods are inclined $(\alpha \neq 0)$. The second component $(\tau)$ is a displacement normal to the vertical plane of the rod inside the bow. Coordinates $z_{d 1}$ and $z_{d 2}$ are directed along the longitudinal axes of the side rods.

The spatial geometry of the side rods causes certain spatial characteristics of their bending. Therefore, their displacement must be considered relative to a three-dimensional Cartesian coordinate system, $O \xi \eta \zeta$. Because the 
side rods are located symmetrically relative to the vertical plane, their displacements are also symmetric. Here they are presented as a sum of two components similar to those of the displacements of a central rod:

$$
\begin{aligned}
& \xi_{d}=\left(x_{V}-z_{d} \sin \alpha\right) \kappa+\left(q_{v} \sin \alpha \cos \beta+q_{\tau} \sin \beta\right) \chi \\
& \eta_{d}=-\left(y_{V}+z_{d} \cos \alpha \cos \beta\right) \kappa+q_{v} \chi \cos \alpha ; \quad \zeta_{d}= \pm\left(q_{v} \sin \alpha \sin \beta-q_{\tau} \cos \beta\right) \chi
\end{aligned}
$$

where $q_{\nu}, q_{\tau}$ are the bending displacements of the free end in the $\nu$ - and $\tau$-directions respectively and $\chi$ is a bending form such as Eq. (2). In Eq. (11) for the lateral displacement component, $\left(\zeta_{d}\right)$, the plus sign in the double sign ' \pm ' is associated with the left-side rod labeled as 1 (Fig. 4), and the minus sign with the right-side rod labeled as 2.

The kinetic energy of the two side rods together is:

$$
T_{d}=\int_{0}^{l_{d}} \rho_{d}\left(\dot{\xi}_{d}^{2}+\dot{\eta}_{d}^{2}+\dot{\zeta}_{d}^{2}\right) d z_{d},
$$

where $l_{d}$ is the length of the side rod and $\rho_{d}$ is the distributed mass of each of the rods.

Substituting Eqs (11) and (2) into Eq. (12), after integration and intermediate transformations:

$$
\begin{aligned}
T_{d}=m_{d}\left\{\begin{array}{l}
\frac{1}{4}\left(9 J_{4}-6 J_{5}+J_{6}\right)\left(\dot{q}_{v}^{2}+\dot{q}_{\tau}^{2}\right)+ \\
{\left[x_{V}^{2}+y_{V}^{2}+2 J_{1} l_{d}\left(y_{V} \cos \alpha \cos \beta-x_{V} \sin \alpha\right)+J_{2} l_{d}^{2}\left(\sin ^{2} \alpha+\cos ^{2} \alpha \cos ^{2} \beta\right)\right] \dot{\kappa}^{2}} \\
+\left[\left(3 J_{2}-J_{3}\right)\left(x_{V} \sin \alpha \cos \beta-y_{V} \cos \alpha\right)-\left(3 J_{3}-J_{4}\right) l_{d} \cos \beta\right] \dot{\kappa} \dot{q}_{v} \\
+\left[\left(3 J_{2}-J_{3}\right) x_{V}-\left(3 J_{3}-J_{4}\right) l_{d} \sin \alpha\right] \dot{\kappa}_{\tau} \sin \beta
\end{array}\right\} \\
+M_{d}\left\{\begin{array}{l}
{\left[\left(x_{V}-l_{d} \sin \alpha\right)^{2}+\left(y_{V}+l_{d} \cos \alpha \cos \beta\right)^{2}\right] \dot{\kappa}^{2}+\dot{q}_{\nu}^{2}+\dot{q}_{\tau}^{2}} \\
+2\left[\left(x_{V}-l_{d} \sin \alpha\right) \sin \alpha \cos \beta-\left(y_{V}+l_{d} \cos \alpha \cos \beta\right) \cos \alpha\right] \dot{\kappa} \dot{q}_{\nu} \\
+2\left[\left(x_{V}-l_{d} \sin \alpha\right) \sin \beta\right] \dot{\kappa} \dot{q}_{\tau}
\end{array}\right\}
\end{aligned}
$$

where $m_{d}=\int_{0}^{l_{d}} \rho_{d} d z_{d}$ is the mass of each rod and $M_{d}$ is the mass of a load concentrated at the free end of the rod.

Because the uni-bar and the extender bar with the handle can be considered as a rigid body, it is possible to model the kinetic energy of the two bars using the expression:

$$
T_{V}=\frac{1}{2} m_{V}\left(\dot{\xi}_{V}^{2}+\dot{\eta}_{V}^{2}\right)+\frac{1}{2} I_{V} \dot{\kappa}^{2}
$$

where $m_{V}$ and $I_{V}$ are the mass and the moment of inertia of the bars relative to their common center of mass with coordinates $x_{V}, y_{V}$. Displacements of this center of mass are defined by the following expressions: $\xi_{V}=x_{V} \kappa$; $\eta_{V}=-y_{V} \kappa$. Substituting these into the previous expression yields the final formula for the kinetic energy of the bars:

$$
T_{V}=\frac{1}{2}\left[m_{V}\left(x_{V}^{2}+y_{V}^{2}\right)+I_{V}\right] \dot{\kappa}^{2} .
$$

\section{Upper and lower rods}

As for other rods, it is possible to write the displacements of the upper and lower rods as a sum of two components:

$$
\xi_{b}=\left(x_{b}+z_{b} \sin \gamma\right) \kappa+q_{b} \chi \sin \gamma ; \quad \eta_{b}=\left(z_{b} \cos \gamma-y_{b}\right) \kappa+q_{b} \chi \cos \gamma ;
$$

where $x_{b}, y_{b}$ are the coordinates of the point where the rod is fixed to the handle; $z_{b}$ is the longitudinal coordinate of the rod; $\gamma$ is the inclination angle relative to the central-rod axis; and $q_{b}$ is the displacement (as a function of time) of the free end of the rod caused by bending. As for previously discussed rods, the kinetic energy of an upper or lower rod can be written in the form:

$$
T_{b}=\frac{1}{2} \int_{0}^{l_{b}} \rho_{b}\left(\dot{\xi}_{b}^{2}+\dot{\eta}_{b}^{2}\right) d z_{b},
$$


where $l_{b}$ is the length of the rod and $\rho_{b}$ is the distributed mass of the rods. Substituting Eqs (15) and (2) into Eq. (16), after integration and intermediate transformations:

$$
\begin{aligned}
T_{b}= & \frac{1}{2} m_{b}\left\{\begin{array}{l}
\frac{1}{4}\left(9 J_{4}-6 J_{5}+J_{6}\right) \dot{q}_{b}^{2}+\left[x_{b}^{2}+y_{b}^{2}+2 J_{1} l_{b}\left(x_{b} \sin \gamma-y_{b} \cos \gamma\right)+J_{2} l_{b}^{2}\right] \dot{\kappa}^{2} \\
+\left[\left(x_{b} \sin \gamma-y_{b} \cos \gamma\right)\left(3 J_{2}-J_{3}\right)+\left(3 J_{3}-J_{4}\right) l_{b}\right] \dot{q}_{b} \dot{\kappa}
\end{array}\right\} \\
& +\frac{1}{2} M_{b}\left\{\begin{array}{l}
{\left[x_{b}^{2}+y_{b}^{2}+l_{b}^{2}+2 l_{b}\left(x_{b} \sin \gamma-y_{b} \cos \gamma\right)\right] \dot{\kappa}^{2}} \\
+\dot{q}_{b}^{2}+2\left[\left(x_{b} \sin \gamma-y_{b} \cos \gamma\right)+l_{b}\right] \dot{q}_{b} \dot{\kappa}
\end{array}\right\}
\end{aligned}
$$

where $m_{b}=\int_{0}^{l_{b}} \rho_{b} d z_{b}$ is the mass of the rod and $M_{b}$ the mass of a load concentrated at the free end of the rod.

\section{Potential energy}

The potential energy of the central and upper / lower rods can be expressed in the context of the technical theory of bending (the Euler-Bernoulli beam) as:

$$
P_{c}=\frac{1}{2} q^{2} \int_{0}^{l c} \varepsilon_{c}\left(\frac{\partial^{2} \chi}{\partial z_{c}^{2}}\right)^{2} d z_{c} \text { and } P_{b}=\frac{1}{2} q_{b}^{2} \int_{0}^{l_{b}} \varepsilon_{b}\left(\frac{\partial^{2} \chi}{\partial z_{b}^{2}}\right)^{2} d z_{b},
$$

where $\varepsilon_{c}$ and $\varepsilon_{b}$ are the distributed stiffness of the rods. Substituting a form of the bending Eq. (2) into this expression yields final expressions for potential energy:

$$
P_{c}=\frac{9 q_{c}^{2}}{2 l_{c}^{4}}\left(1-2 J_{\varepsilon 1 c}+J_{\varepsilon 2 c}\right) \int_{0}^{l c} \varepsilon_{c} d z_{c} ; \quad P_{b}=\frac{9 q_{b}^{2}}{2 l_{b}^{4}}\left(1-2 J_{\varepsilon 1 b}+J_{\varepsilon 2 b}\right) \int_{0}^{l_{b}} \varepsilon_{b} d z_{b},
$$

where

$$
J_{\varepsilon i c}=\frac{\int_{0}^{l c} \varepsilon_{c} z^{i} d z_{c}}{l_{c}^{i} \int_{0}^{l c} \varepsilon_{c} d z_{c}} \text { and } J_{\varepsilon i b}=\frac{\int_{0}^{l_{b}} \varepsilon_{b} z^{i} d z_{b}}{l_{b}^{i} \int_{0}^{l_{b}} \varepsilon_{b} d z_{b}}
$$

are nondimensional parameters of the cross section of the rods relative to its bending.

On the other hand, because the form of the bending equation was borrowed from the problem of the bending of a horizontal cantilever beam with a concentrated force at the free end [10], it is possible to express the potential energy as:

$$
P_{c}=\frac{1}{2} c_{c} q^{2} \text { and } P_{b}=\frac{1}{2} c_{b} q_{b}^{2},
$$

where $c_{c}$ and $c_{b}$ are the stiffness of the rods relative to a normal force at the free end. Based on Eq. (18), the stiffness can be expressed as:

$$
c_{c}=\frac{9}{l_{c}^{4}}\left(1-2 J_{\varepsilon 1 c}+J_{\varepsilon 2 c}\right) \int_{0}^{l c} \varepsilon_{c} d z_{c}
$$

A similar expression can be developed for the stiffness of an upper or lower rod:

$$
c_{b}=\frac{9}{l_{b}^{4}}\left(1-2 J_{\varepsilon 1 b}+J_{\varepsilon 2 b}\right) \int_{0}^{l_{b}} \varepsilon_{b} d z_{b} .
$$

In the same way, expressions for the potential energy of the side rods together and for the upper and lower rods can be obtained: 


$$
P_{d}=c_{d}\left(q_{\nu}^{2}+q_{\tau}^{2}\right)
$$

as well as stiffness expressions for each of them:

$$
c_{d}=\frac{9}{l_{d}^{4}}\left(1-2 J_{\varepsilon 1 d}+J_{\varepsilon 2 d}\right) \int_{0}^{l_{d}} \varepsilon_{d} d z_{d} .
$$

A modular displacement vector for the bending of the free end of a side rod can be defined as:

$$
q_{d}=\sqrt{q_{\nu}^{2}+q_{\tau}^{2}}
$$

and its direction can be defined as $\lambda=\operatorname{arctg} \frac{q_{\tau}}{q_{\nu}}$.

Using the expressions for the kinetic and potential energy of a bow and arrow [10], it is possible to develop a model of the recoil and vibration of a bow-and-stabilizer system.

\section{Recoil and vibration model of a bow-and-stabilizer system}

Rigorous three-dimensional analysis of the space system is very complicated and with the other assumptions is not essential. The main part of potential energy, stored in bow limbs, transfers to kinetic energy of the longitudinal motion of the arrow and the string joint motion. Some negligible part of it transfers to the deflect motion of the arrow. Although the arrow is in a space motion of the whole system, the problem can be idealized and reduced with two separate systems. The first one is in the vertical plane and the second is in the lateral plane [11].

Consider a common design for a stabilizer with cylindrical rods: $\rho=$ const, $\varepsilon=$ const. Substituting Eqs (10), (13), (14), (17), (19), and (22) and the expressions for the kinetic and potential energy of a bow and arrow [10] into the Lagrange equations of the second kind:

$$
\frac{d}{d t}\left(\frac{\partial T}{\partial \dot{v}}\right)-\frac{\partial T}{\partial v}+\frac{\partial P}{\partial v}=0
$$

the result is a system of differential equations relative to a set of generalized coordinates $v \equiv \kappa, \theta_{U}, \theta_{L}, q_{c}, q_{\nu}, q_{\tau}, q_{b}$, $\xi_{A}, \eta_{A}, \psi$ (Fig. 5):

$$
\begin{aligned}
& \left\{\begin{array}{l}
I_{H}+I_{U}+I_{L}+m_{U} h_{U}^{2}+m_{L} h_{L}^{2}+I_{V}+m_{V}\left(x_{V}^{2}+y_{V}^{2}\right) \\
+I_{c m}+I_{d m}+I_{b m}+I_{c M}+I_{d M}+I_{b M}
\end{array}\right\} \ddot{\kappa} \\
& +m_{U} r_{U} h_{U}\left[b_{1}\left(\ddot{\theta}_{U}+2 \ddot{\kappa}\right)-b_{2}\left(\ddot{\theta}_{U}+\ddot{\kappa}\right)^{2}\right]- \\
& m_{L} r_{L} h_{L}\left[b_{3}\left(\ddot{\theta}_{L}-2 \ddot{\kappa}\right)-b_{4}\left(\ddot{\theta}_{L}-\ddot{\kappa}\right)^{2}\right]+I_{U} \ddot{\theta}_{U}-I_{L} \ddot{\theta}_{L} \\
& +2 m_{d}\left(Q_{\nu m} \ddot{q}_{\nu}+Q_{\tau m} \ddot{q}_{\tau}\right)+2 M_{d}\left(Q_{\nu M} \ddot{q}_{\nu}+Q_{\tau M} \ddot{q}_{\tau}\right)+ \\
& \left(m_{c} Q_{c m}+M_{c} Q_{c M}\right) \ddot{q}_{c}+\left(m_{b} Q_{b m}+M_{b} Q_{b M}\right) \ddot{q}_{b}+ \\
& e_{U}\left[S_{2}\left(b_{1} l_{U}+h_{U}\right)-S_{1} b_{2} l_{U}\right]-e_{L}\left[S_{4}\left(b_{3} l_{L}+h_{L}\right)+S_{3} b_{4} l_{L}\right]=0 ; \\
& I_{U}\left(\ddot{\theta}_{U}+\ddot{\kappa}\right)+m_{U} r_{U} h_{U} b_{1} \ddot{\kappa}+c_{U}\left(\theta_{U}+\varphi_{U}\right)+e_{U} l_{U}\left(S_{U \xi} b_{1}-S_{U \eta} b_{2}\right)=0 ; \\
& I_{L}\left(\ddot{\theta}_{L}-\ddot{\kappa}\right)-m_{L} r_{L} h_{L} b_{3} \ddot{\kappa}+c_{L}\left(\theta_{L}+\varphi_{L}\right)+e_{L} l_{L}\left(S_{L \xi} b_{3}+S_{L \eta} b_{4}\right)=0 ; \\
& \left(\frac{33}{140} m_{c}+M_{c}\right) \ddot{q}_{c}+\left(m_{c} Q_{c m}+M_{c} Q_{c M}\right) \ddot{\kappa}+c_{c} q_{c}=0 ; \\
& \left(\frac{33}{140} m_{d}+M_{d}\right) \ddot{q}_{\nu}+\left(m_{d} Q_{\nu m}+M_{d} Q_{\nu M}\right) \ddot{\kappa}+c_{d} q_{\nu}=0 ;
\end{aligned}
$$




$$
\begin{aligned}
& \left(\frac{33}{140} m_{d}+M_{d}\right) \ddot{q}_{\tau}+\left(m_{d} Q_{\tau m}+M_{d} Q_{\tau M}\right) \ddot{\kappa}+c_{d} q_{\tau}=0 ; \\
& \left(\frac{33}{140} m_{b}+M_{b}\right) \ddot{q}_{b}+\left(m_{b} Q_{b m}+M_{b} Q_{b M}\right) \ddot{\kappa}+c_{b} q_{b}=0 ; \\
& \left(m_{A}+m_{a}\right) \ddot{\xi}_{A}-e_{U} S_{U \xi}-e_{L} S_{L \xi}=0 ; \quad\left(m_{A}+m_{a}\right) \ddot{\eta}_{A}+m_{a} r_{A} \ddot{\psi}+m_{a} g-e_{U} S_{U \eta}-e_{L} S_{L \eta}=0 ; \\
& I_{A} \ddot{\psi}+m_{a} r_{A}\left(\ddot{\eta}_{A}+\ddot{\xi}_{A} \psi+g\right)=0,
\end{aligned}
$$

where

$$
\begin{aligned}
& I_{c m}=m_{c}\left(\frac{l_{c}^{2}}{3}+x_{V}^{2}+y_{V}^{2}-y_{V} l_{c}\right) ; Q_{c m}=\frac{11}{40} l_{c}-\frac{3}{4} y_{V} ; I_{c M}=M_{c}\left[x_{V}^{2}+\left(l_{c}-y_{V}\right)^{2}\right] ; \\
& Q_{c M}=l_{c}-y_{V} ; I_{b m}=m_{b}\left(x_{b}^{2}+y_{b}^{2}+l_{b} r_{b}+\frac{1}{3} l_{b}^{2}\right) ; Q_{b m}=\frac{3}{8} r_{b}+\frac{11}{40} l_{b} ; r_{b}=x_{b} \sin \gamma-y_{b} \cos \gamma ; \\
& I_{b M}=M_{b}\left(x_{b}^{2}+y_{b}^{2}+2 l_{b} r_{b}+l_{b}^{2}\right) ; \quad Q_{b M}=r_{b}+l_{b} ; \\
& I_{d m}=2 m_{d}\left[x_{V}^{2}+y_{V}^{2}+l_{d}\left(y_{V} \cos \alpha \cos \beta-x_{V} \sin \alpha\right)+\frac{1}{3} l_{d}^{2}\left(\sin ^{2} \alpha+\cos ^{2} \alpha \cos ^{2} \beta\right)\right] ; \\
& I_{d M}=2 M_{d}\left[\left(x_{V}-l_{d} \sin \alpha\right)^{2}+\left(y_{V}+l_{d} \cos \alpha \cos \beta\right)^{2}\right] ; \\
& Q_{\nu m}=\frac{3}{8}\left(x_{V} \sin \alpha \cos \beta-y_{V} \cos \alpha\right)-\frac{11}{40} l_{d} \cos \beta ; \quad Q_{\tau m}=\left(\frac{3}{8} x_{V}-\frac{11}{40} l_{d} \sin \alpha\right) \sin \beta ; \\
& Q_{\nu M}=x_{V} \sin \alpha \cos \beta-y_{V} \cos \alpha-l_{d} \cos \beta ; \quad Q_{\tau M}=\left(x_{V}-l_{d} \sin \alpha\right) \sin \beta ; \\
& e_{U}=\frac{f\left(s_{U}-S_{U}\right)}{s_{U} S_{U}} ; e_{L}=\frac{f\left(s_{L}-S_{L}\right)}{s_{L} S_{L}} ; \quad s_{U}=\sqrt{S_{1}^{2}+S_{2}^{2}} ; s_{L}=\sqrt{S_{3}^{2}+S_{4}^{2}} ; \\
& S_{1}=h_{U}+l_{U} b_{1}-\eta_{A} ; \quad S_{2}=h_{U} \kappa+l_{U} b_{2}-\xi_{A} ; \quad S_{3}=h_{L}+l_{L} b_{3}+\eta_{A} ; \quad S_{4}=h_{L} \kappa-l_{L} b_{4}+\xi_{A} ; \\
& b_{1}=\cos \left(\theta_{U}+\kappa\right) ; b_{2}=\sin \left(\theta_{U}+\kappa\right) ; \quad b_{3}=\cos \left(\theta_{L}-\kappa\right) ; b_{4}=\sin \left(\theta_{L}-\kappa\right) ;
\end{aligned}
$$

$l_{U}, l_{L}$ are the lengths of the limbs; $s_{U}, s_{L}$ are the lengths of the string segments in the drawn-bow situation; $S_{U}, S_{L}$ are the lengths of the segments of the free string; $h_{U}, h_{L}$ are the virtual lengths of the riser, i.e., the distances from the pivot point $(O)$ to the points of the virtual elastic elements of the limbs; $c_{U}, c_{L}$ are the stiffness values of the limbs; $l_{a}$ is the length of the arrow (and also its drawn distance); $f$ is a stiffness parameter of the string; is the mass of the string attached to the nock point; $m_{s}$ is the mass of the string; $m_{a}$ is the mass of the arrow; $\psi$ is the inclination angle of the arrow; $I_{H}$ is the moment of inertia of the riser relative to the pivot point; are the masses of the limbs with the added mass of the string $\left(1 / 3 m_{s}\right)$ attached to the tips; $I_{U}, I_{L}$ are the moments of inertia of limbs relative to their junction points with the riser, with the addition of the same portion of the string mass; $r_{U}, r_{L}$ are the distances from the mass centers of the limbs to their junction points; and $g$ is the acceleration due to gravity. The subscripts "U" and "L" correspond to the upper and lower limbs.

The handle, arrow and stabilizer were described using linear models. Correspondent part of the dynamic model was linear too, e.g. terms in Eq. (25). Because displacements of limbs are commensurable with dimensions of a bow, a non-linear model was applied. Correspondent terms in Eq. (25) are non-linear.

The initial conditions of the problem are:

$$
\begin{aligned}
t & =0, \xi_{A}=\xi_{A 0} ; \eta_{A}=\eta_{A 0} ; \theta_{U}=\theta_{U 0} ; \theta_{L}=\theta_{L 0} ; \kappa=0 ; \psi=\psi_{0} ; q_{c}=0 ; q_{\nu}=0 ; \\
q_{\tau} & =0 ; \dot{\xi}_{A}=0 ; \dot{\eta}_{A}=0 ; \dot{\theta}_{U}=0 ; \dot{\theta}_{L}=0 ; \dot{\kappa}=0 ; \dot{\psi}=0 ; \dot{q}_{c}=0 ; \dot{q}_{\nu}=0 ; \dot{q}_{\tau}=0,
\end{aligned}
$$

where the constants $\eta_{A 0}, \theta_{U 0}, \theta_{L 0}$ are solutions of the static problem [5]. Zero values of the derivatives correspond to sport archery practice, i.e., breathing is stopped, and the archer is motionless. 


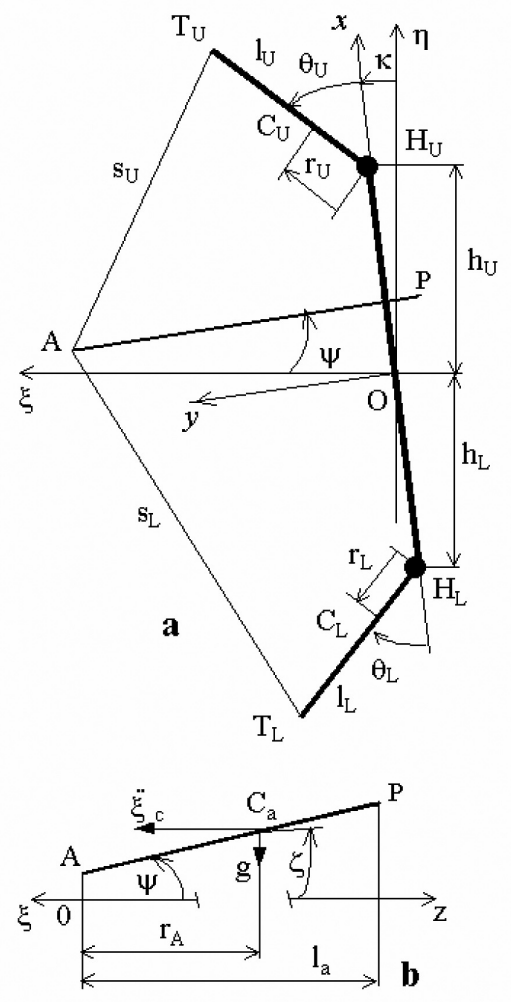

Fig. 5. (a) Dynamic schematic model of a bow; (b) schematic model of an arrow.

\section{Numerical example}

Consider a modern sport bow with parameters as in [4]: $l_{U}=l_{L}=531 \mathrm{~mm} ; m_{U}=m_{L}=0.107 \mathrm{~kg} ; I_{U}=I_{L}=$ $68.2 \mathrm{~kg}-\mathrm{cm}^{2} ; r_{U}=r_{L}=228 \mathrm{~mm} ; c_{U}=c_{L}=69.1 \mathrm{Nm} ; \varphi_{U}=0.605 ; \varphi_{L}=0.608 ; h_{U}=h_{L}=342 \mathrm{~mm} ; I_{H}=$ $2130 \mathrm{~kg}-\mathrm{cm}^{2} ; S_{U}=78 \mathrm{~cm} ; S_{L}=84 \mathrm{~cm} ; f=25515 \mathrm{~N} ; m_{s}=6.9 \mathrm{~g}$.

From the static problem described in [5], $\eta_{A 0}=43 \mathrm{~mm} ; \theta_{U 0}=0,766 ; \theta_{L 0}=0,794$. The rest point is located at $\eta_{P 0}=43 \mathrm{~mm}$.

The parameters of the arrow are: $l_{a}=783 \mathrm{~mm} ; m_{a}=22.4 \mathrm{~g} ; I_{A}=73.6 \mathrm{~kg}-\mathrm{cm}^{2} ; r_{A}=510 \mathrm{~mm}$.

The parameters of the stabilizer are [1]: $m_{c}=0.193 \mathrm{~kg} ; m_{b}=0.088 \mathrm{~kg} ; m_{d}=0.065 \mathrm{~kg} ; x_{V}=-107 \mathrm{~mm}$; $y_{V}=-142 \mathrm{~mm} ; x_{b}=221 \mathrm{~mm} ; y_{b}=-18 \mathrm{~mm} ; m_{V}=0.119 \mathrm{~kg} ; I_{V}=21 \mathrm{~kg}-\mathrm{cm}^{2} ; c_{c}=485 \mathrm{Nm}^{-1} ; c_{b}=$ $833 \mathrm{Nm}^{-1} ; c_{d}=1170 \mathrm{Nm}^{-1} ; l_{c}=760 \mathrm{~mm} ; l_{b}=420 \mathrm{~mm} ; l_{d}=250 \mathrm{~mm} ; \alpha_{\max }=0.297 \mathrm{rad}\left(17^{\circ}\right) ; \beta_{\max }=$ $0.617 \mathrm{rad}\left(35^{\circ}\right) ; \gamma=0.157 \mathrm{rad}\left(9^{\circ}\right) ; M_{c}=0.043 \mathrm{~kg} ; M_{b}=0.037 \mathrm{~kg} ; M_{d}=0.028 \mathrm{~kg}$.

The system of Eq. (25) with initial conditions (26) represents the Cauchy problem for second-order ordinary differential equations. It is impossible to obtain an analytical solution for this problem, and therefore the Runge-Kutta method as implemented in the NDSolve module of the Mathematica software package was used.

During joint movement of the arrow and string, the central rod bends upward in the vertical plane of symmetry and describes approximately one-fifth of a circle of vibration. The maximum displacement of its free end is approximately $1 \mathrm{~mm}$ (Fig. 6). This figure illustrates the process of bow stabilization in the vertical plane during joint motion of the bow and arrow. String-and-arrow joint motion (internal ballistic motion) is accompanied by intensive vibration, which is caused by destruction of the static balance of forces at the moment of string release [6]. The clockwise angular displacement of the bow riser is partly compensated for by counterclockwise bending of the central rod with a 1.2-mm displacement of its free end. The monotonic character of these movements reveals that this process takes place below the resonant frequency of the system.

Simultaneously with the central rod, the side rods vibrate, approximately describing two circles. Independently of side-rod position, the characteristics of the vibration are the same. The first displacement maximum appears at 
Table 2

Module displacements of the free ends of a stabilizer side rod $\left(\mathrm{m}^{-6}\right)$

\begin{tabular}{lcccccccc}
\hline Rotation & $\beta$ & \multicolumn{3}{c}{$\alpha=0^{\circ}$} & & \multicolumn{3}{c}{$\alpha=17^{\circ}$} \\
\cline { 3 - 5 } \cline { 6 - 8 } points & & $q$ & $q$ & $q_{d}$ & & $q$ & $q$ & $q_{d}$ \\
\hline $\max -1$ & $0^{\circ}$ & 2.8 & 0 & 2.8 & & 5.2 & 0 & 5.2 \\
$\min$ & & 0.7 & 0 & 0.7 & & 1,4 & 0 & 1.4 \\
$\max -2$ & & 2.1 & 0 & 2.1 & & 4,0 & 0 & 4.0 \\
$\max -1$ & $35^{\circ}$ & 5.1 & 4.2 & 6.6 & & 2.6 & 6.2 & 6.7 \\
$\min$ & & 1.3 & 1.1 & 1.7 & & 0.7 & 1.6 & 1.7 \\
$\max -2$ & & 3.9 & 3.2 & 5.0 & & 2.0 & 4.9 & 5.3 \\
\hline
\end{tabular}
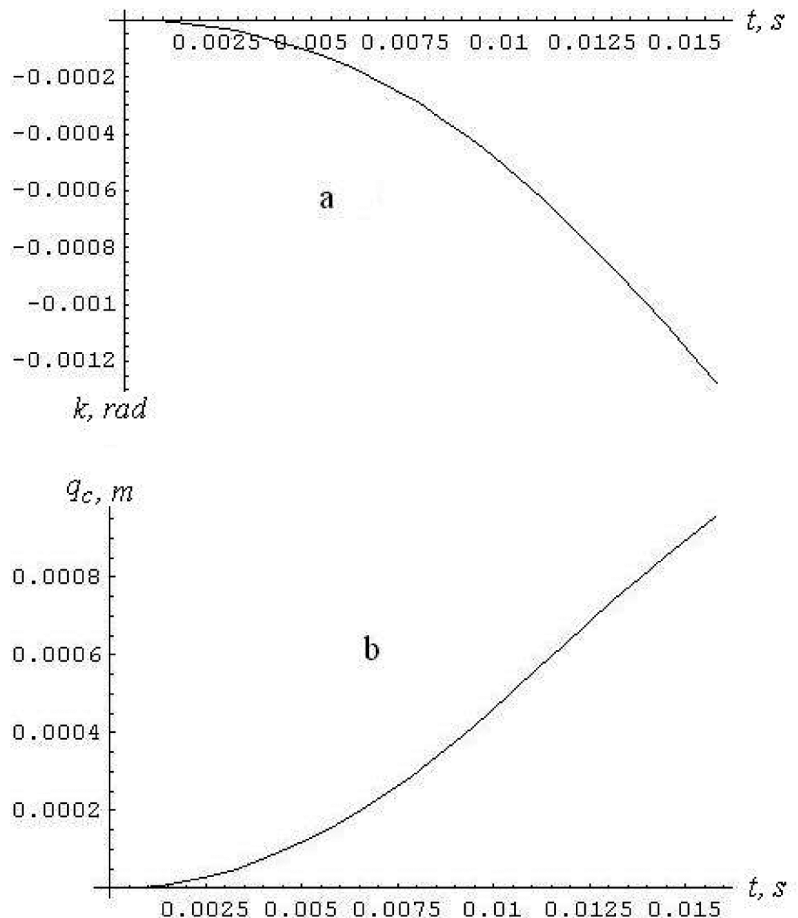

Fig. 6. (a) Angle of rotation of a stabilizer with a riser; (b) bending displacement of the free ends of the central rod.

$4.6 \mathrm{~s}$ after string release (Fig. 7). The first minimum appears at $9.4 \mathrm{~ms}$ and the second maximum at $12.7 \mathrm{~ms}$. The total time of internal ballistic motion is $15.8 \mathrm{~ms}$ and does not depend (within calculation accuracy) on the side-rod positions. However, the bending displacements of the side rods do depend significantly on their position. The maximum extension in a normal direction to the three turning points mentioned $\left(q_{\nu}=5.2 ; 1.4 ; 4.0\right)$ occurs when the side rods are parallel $(\beta=0)$ and have a maximum angle of inclination $\left(\alpha=17^{\circ}\right)$ with the central rod (Table 2$)$. The tangential components are at a maximum $\left(q_{\tau}=6.2 ; 1.6 ; 4.9\right)$ when the side-rod angle of separation is at a maximum $\left(\beta=35^{\circ}\right)$ and the side rods have a maximum angle of inclination $\left(\alpha=17^{\circ}\right)$. It was easy to predict that there are no tangential components $\left(q_{\tau}=0\right)$ when the side rods are parallel $(\beta=0)$, independently of their inclination. The maximum module extension occurs when the side rods are at their greatest angle of separation.

One useful function of a stabilizer to accumulate a portion of the bow vibration energy in the bending of its rods. A quantitative parameter describing this process is the displacement of the rods. As can be seen from computer simulation, bending vibration is highly dependent on the position of the rods. Other factors being equal, the vibration amplitude of the side rods increases with their angle of separation. When the side rods are at their maximum separation, the module extension is 1.3 times greater (for maximum inclination) and 2.4 times greater (for zero inclination) than when the side rods are parallel.

Because of the bending of the rods, energy dissipation occurs in the stabilizer. A quantitative parameter describing this process is the decrement of dissipation, $\delta=\ln \frac{q_{1}}{q_{2}}$, where $q_{1}, q_{2}$ are the amplitudes of subsequent circles of 

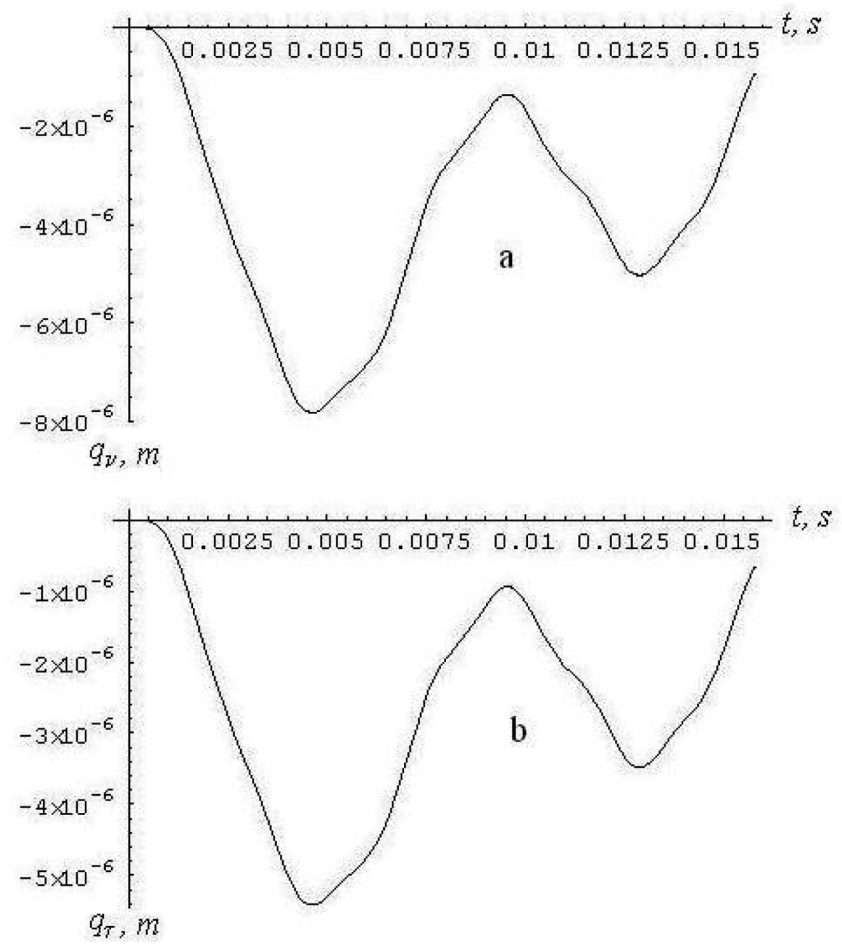

Fig. 7. Normal (a) and tangential (b) components of the displacement of the free ends of the stabilizer side rods (with angle of rod inclination $\alpha=17^{\circ}$ and separation angle $\beta=35^{\circ}$ ).

vibration. The decrement of dissipation measures the speed of damping of vibration. A parameter which varies inversely to the decrement is the number of circles required to decrease the amplitude $e \approx 2.7$ times. Taking into account the first and second maxima (Fig. 7), in the computer simulation results, $\delta=0.24-0.29$. This number shows the high level of energy dissipation that occurs in these side rods, because in the theory of vibrations for resonant systems, a sufficiently large value of the decrement of dissipation is considered to be 0.1 . In effect, during three or four circles of the side rods, i.e., $0.028-0.034 \mathrm{~s}$, the amplitude of vibration decreases by an order of magnitude.

According to the properties of the damping model, the dissipation power is proportional to the square of the speed of deformation (14), i.e., to the square of the amplitude when frequencies are equal. This means that when the side rods are at their maximum separation and maximum deflection, the maximum amount of vibration is being dissipated in these rods. As more vibration energy dissipates from the rods, less vibration energy remains in the bow riser to act impulsively on the archer. This makes it possible to increase the damping power of a stabilizer by increasing the separation of the side rods and decreasing the inclination of their plane. When the side rods have maximum inclination and maximum separation, they achieve 1.7 times more energy dissipation than do parallel rods. When side rods are installed with maximum separation and zero inclination, the energy dissipation is increased 5.6 times.

Kinetic energy of the handle and limbs during the string and arrow joint motion when there is no stabilizer on the bow $(T)$ is $79 \%$ greater (Fig. 8) relatively the same bow which is equipped with a multi-rod stabilizer ( $T *$ ). There are seven full cycles of oscillation during the motion.

\section{Conclusions}

The common motion of a bowstring and arrow (internal ballistic motion) is accompanied by intense vibration, which is caused by destruction of the static balance of forces at the moment of string release. The clockwise angular motion of the bow handle is partly compensated for by counterclockwise bending of the central rod with displacement 

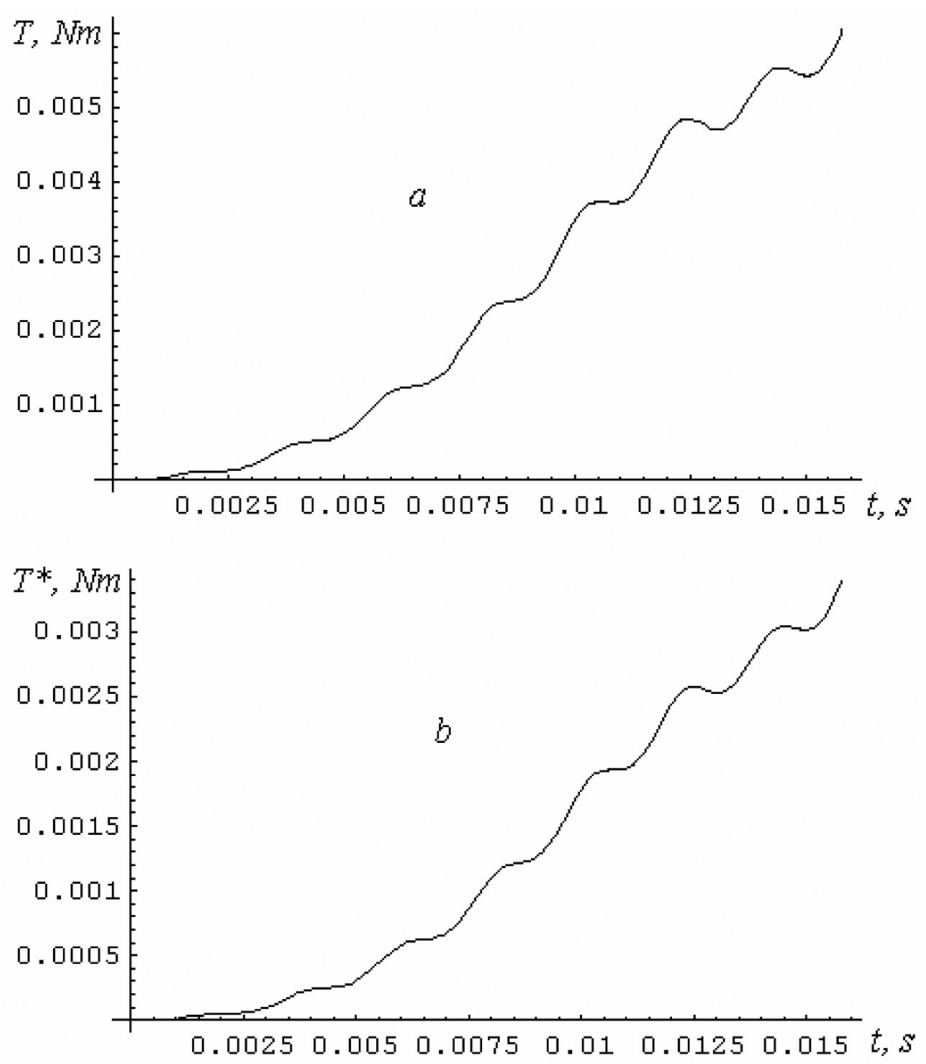

Fig. 8. Kinetic energy of the handle and limbs during string and arrow joint motion when there is no stabilizer on the bow (a) and when the same bow is equipped with a multi-rod stabilizer (b).

of its free end. The monotonic nature of these movements reveals that this process is operating below the resonant frequency of the system.

Kinetic energy of the handle and limbs during the string and arrow joint motion when there is no stabilizer on the bow is $79 \%$ greater relatively the same bow which is equipped with a multi-rod stabilizer. There are seven full cycles of oscillation during the motion.

The side rods vibrate by making approximately two circles. Independently of side-rod position, the characteristics of this vibration are the same: two local maxima and one local minimum at the same points in time. In an effort to reduce the disagreeable recoil and vibration of the bow during the time of internal ballistic motion, it is possible to increase (by 1.7-5.6 times) the damping power of the stabilizer by increasing the separation of the side rods and decreasing the inclination of their plane.

Two further problems arise from the results of this research which are significant for archery training and competition with regard to bow recoil and vibration. The model proposed here should be enhanced by a model of the properties of the archer's body. The second problem is to study the vibration of a bow after an arrow has been launched from the string.

\section{Acknowledgment}

1. The research was partly supported by NATO Scientific Affairs Division (Collaborative Linkage Grant \#LST.CLG.977859). The support of the Division is gratefully acknowledged.

2. Anonymous Reviewers are gratefully acknowledged for their fruitful comments. 


\section{Appendix 1}

Easton $X 10^{\mathrm{TM}}$ Stabilizer System [1]
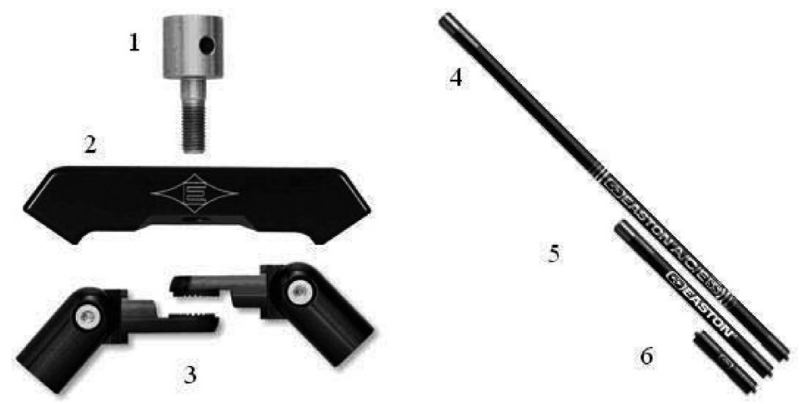

Fig. A1. Stabilizer System: 1 - fastening bolt; 2 - uni-bar; 3 - side-rod adjustable members; 4 - central rod; 5 - side rod; 6 - extender bar.

\section{Appendix 2}

Mass inertial parameters of stabilizer rods:

$$
\begin{aligned}
& \int_{0}^{l_{c}} \rho_{c} \chi d z_{c}=\frac{1}{2} m_{c}\left(3 J_{2}-J_{3}\right) ; \quad \int_{0}^{l_{c}} \rho_{c} \chi d z_{c}=\frac{1}{2} m_{c} l_{c}\left(3 J_{3}-J_{4}\right) ; \int_{0}^{l_{c}} \rho_{c} \chi^{2} d z=\frac{1}{4} m_{c}\left(9 J_{4}-6 J_{5}+J_{6}\right) ; \\
& \int_{0}^{l_{c}} \rho_{c} \chi d z_{c}=\frac{1}{2} m_{c}\left(3 J_{2}-J_{3}\right) ; \quad \int_{0}^{l_{c}} \rho_{c} \chi z_{c} d z_{c}=\frac{1}{2} m_{c} l_{c}\left(3 J_{3}-J_{4}\right) ; \\
& \int_{0}^{l} \rho \chi^{2} d z=\frac{1}{4} m\left(9 J_{4}-6 J_{5}+J_{6}\right)=\frac{33}{140} m ; \int_{0}^{l_{c}} \rho_{c} z_{c} d z_{c}=m_{c} l_{c} J_{1} ; \int_{0}^{l_{c}} \rho_{c} z_{c}^{2} d z_{c}=m_{c} l_{c}^{2} J_{2} .
\end{aligned}
$$

\section{References}

[1] Easton X10 ${ }^{\mathrm{TM}}$ Stabilizer System, http://www.eastonarchery.com (2009).

[2] S. Ellison, Controlling Bow Behaviour with Stabilisers. http://www.tenzone.u-net.com/Equipment/stabilisation/pdfs/stab4a4.pdf, 2009.

[3] A.N. Kalinichenko, Influence of human factors on functioning of the archer-bow system, Theory and Methods of Physical Education 6 (2008), 12-17 (in Ukrainian).

[4] WIN\&WIN Recurve Bow, http://www.win\&win.com (2009).

[5] I. Zaniewski, Archer-bow-arrow behavior in the vertical plane, Acta of Bioengineering and Biomechanics 8 (2006), 65-82.

[6] I. Zaniewski, Bow tuning in the vertical plane, Sports Engineering 9 (2006), 77-86.

[7] I. Zaniewski, Dynamics of arrow-bow system, Journal of Automation and Information Sciences 31 (1999), 11-17.

[8] I. Zaniewski, Lateral deflection of archery arrows, Sports Engineering 4 (2001), 23-42.

[9] I. Zaniewski, Mechanical and mathematical modeling of sport archery arrow ballistics, International Journal of Computer Science in Sport 7 (2008), 37-46.

[10] I. Zaniewski, Modeling and computer simulation of bow stabilization in the vertical plane, International Journal of Sports Science and Engineering 2 (2008), 3-14.

[11] I. Zaniewski, Modeling of the archery bow and arrow vibrations, Shock and Vibration 16 (2009), 307-317. 

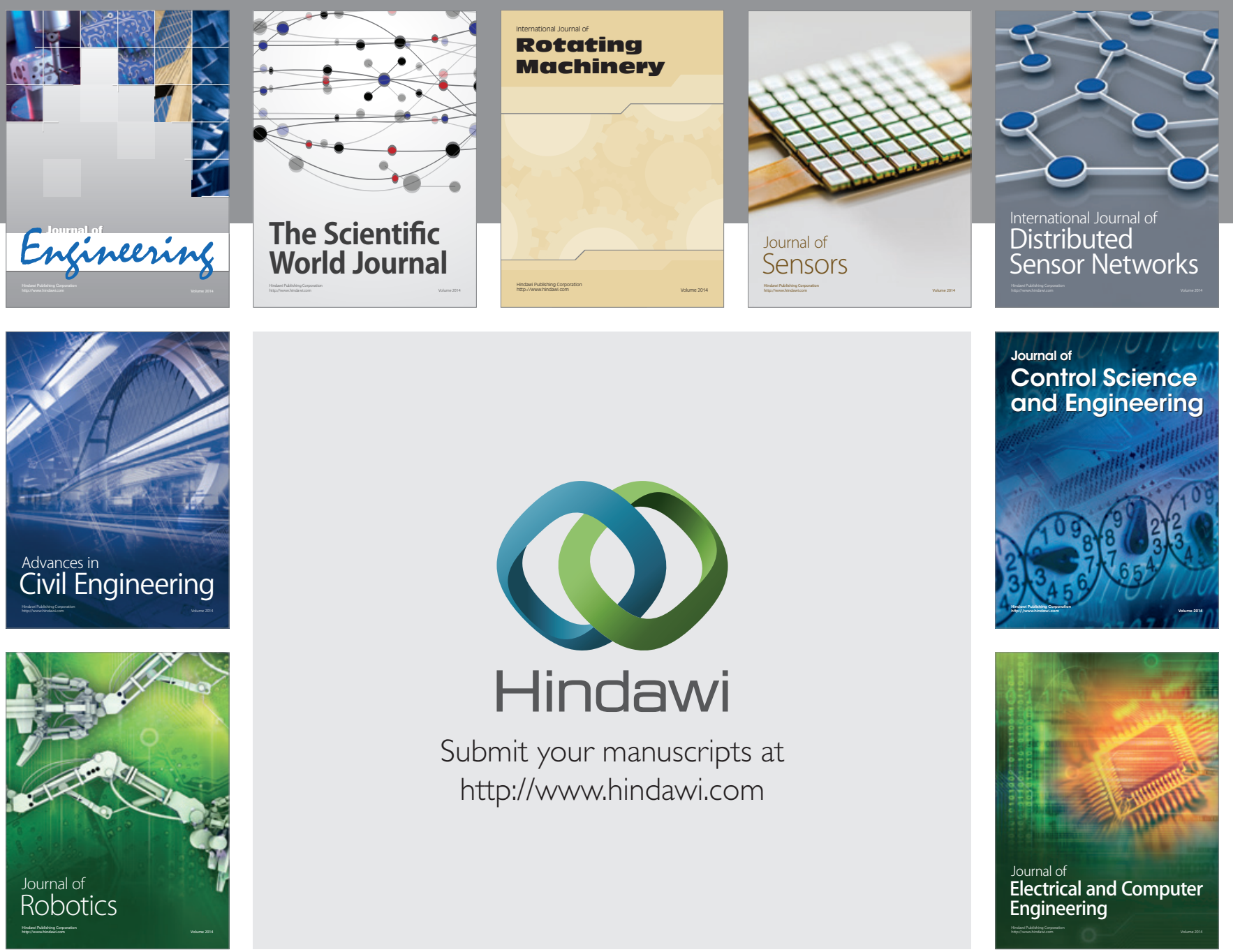

Submit your manuscripts at

http://www.hindawi.com
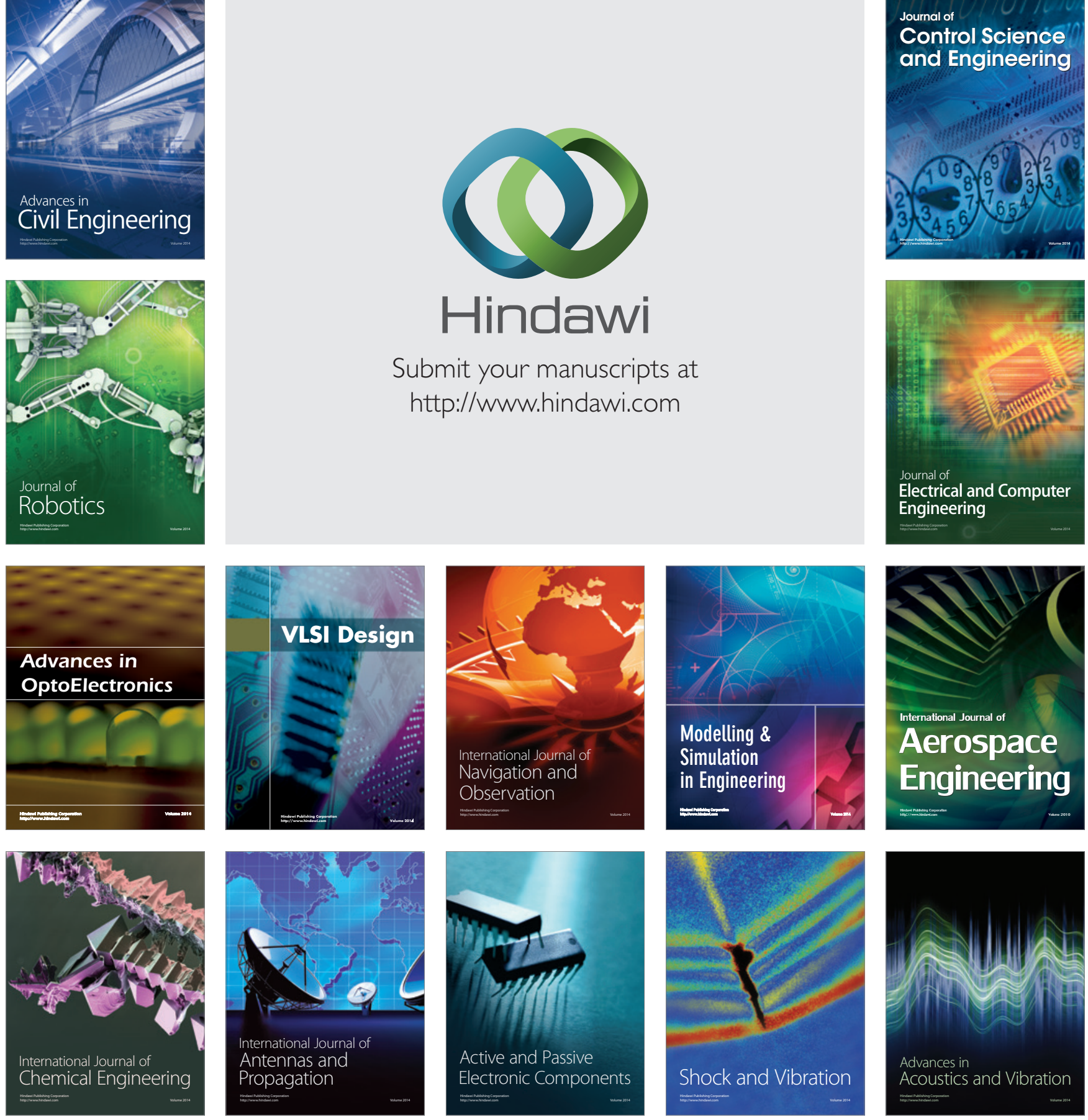